\title{
Nano Computing: An Introduction
}

\author{
Matthew N. O. Sadiku, Chandra M. M. Kotteti, and Sarhan M. Musa \\ Roy G. Perry College of Engineering \\ Prairie View A\&M University \\ Prairie View, TX 77446 \\ U.S.A
}

\begin{abstract}
Nanocomputing refers to computing devices built from nanoscale components. It is an emerging technology that is at the early stage of its development. An exciting anticipation of nanocomputing is the smaller system size it will provide and the ability to construct systems that use many orders of magnitude more components than in the past. This paper provides a brief introduction to nanocomputing.
\end{abstract}

Key words: Nano Computing, Nanotechnology.

\section{INTRODUCTION}

We have witnessed tremendous speed up in computing power. We are approaching the end of an era in computing: the era where Moore's Law reigns, where processing power per dollar doubles every year. The laws of quantum mechanics and the limitations of fabrication techniques soon will prevent further reduction in the minimum size of today's semiconductor components. As the IC industry started building commercial devices at such size scales in the 2000s, the term "nanocomputing" has been reserved for device features of only a few nm.

Nanocomputing describes computing that uses extremely tiny or nanoscale (one nanometer [nm] is one billionth of a meter) devices. It relates to computing systems which are constructed from nanoscale components. The goal of nanocomputing is to deliver computers smaller than 10 nanometers. Nanocomputing is based on nanotechnology.

\section{NANOTECHNOLOGY}

Nanotechnology is the engineering of systems at the molecular or nano scale. It has been called the second Industrial Revolution because of the special properties of materials at the nanoscale. It is a branch of green technology which has the potential to revolutionize many aspects of our lives. It has permeated all sectors of our economy due to the unique properties of materials at the nanoscale. It is transforming the world of materials and its influence will be broad. It will not only initiate the next industrial revolution, it will offer technological solutions.

Richard Feynman, a famous Nobel Laureate physicist, is regarded as the father of nanotechnology. Nanotechnology has provided effective processing features, miniaturization from micro to nano obeying the Moore's law, which states that CPU is doubled in performance every 18 months [1]. Nanotechnology is the science of small things-at the atomic level or nanoscale level. It has the idea that the technology of the future will be built on atoms. It has impact on every area of science and technology $[2]$.

Nanotechnology involves imaging, measuring, modeling, and manipulating matter at the nano scale. It covers a wide variety of disciplines like physics, chemistry, biology, biotechnology, information technology, engineering, and their potential applications. Nanotechnology has a vast range of applications, such as in nanomedicine, nanoelectronics, biomaterials energy production, and consumer products. It is revolutionizing many industry sectors: information technology, homeland security, medicine, transportation, energy, food safety, and environmental science. Nanoparticles are used increasingly in catalysis to boost chemical reactions, especially $\mathrm{n}$ petroleum refining and automotive catalytic converters. Nanotechnology will likely revolutionize sensor capabilities. Nanosensors and nanoactuators have the potential for improving the capability of sensor networks. 


\section{CONCEPT OF NANOCOMPUTING}

Nanotechnology has laid its path in nanocomputing leading towards the solutions for a complex problems. Nanocomputers are electronic computers that contain components with dimensions of only a few nanometers and deal with materials at a molecular level. They may include massive number of devices. They are likely to be up to 10,000 times more densely integrated than today's smallest microcomputers. They can be orders of magnitude faster than current electronic computers.

With fast-moving nanotechnology, nanocomputers will eventually scale down to the atomic level and will be measured in nanometers. As an alternative to using transistor, nanocomputers might have an entirely new type of circuits or architecture made up of many simple units called cells. Such circuits would consume far less power, increasing battery life and shrinking boxes and fans necessary to cool circuits.

Nanocomputing can be produced by a number of nanoscale structures such as DNA and proteins. Nanocomputers can be built in many ways leading to different kinds of nanocomputers [1,3]:

- Electronic Nanocomputing: Nanocomputers can be electronic, where nanolithography is used to create microscopic circuits. These would operate in a manner similar to the way present-day microcomputers work. Nanoelectronics-based intelligent sensors provide a unique opportunity to interface with the physical world at the submicron scale and below. Some see electronic nanocomputers to be most likely future computers.

- Mechanical Nanocomputing: This focusses on the microscopic moving parts and works on a purely mechanical basis. Mechanical nanocomputers would use tiny moving components called nanogears to encode information. Nanorobots can operate or be controlled by mechanical nanocomputers. However, mechanical nanocomputer technology has sparked controversy and some consider it unworkable.

- Chemical/Biochemical Nanocomputing: A chemical computer is one that processes information by making and breaking chemical bonds. It processes and stores information in the form of chemical structures and their interactions. Both chemical and biochemical nanocomputers would store and process information in terms of chemical structures and interactions.

- Quantum Nanocomputing: The quantum nanocomputers rely on quantum physics. They are planned to hold each bit of data as a quantum state of the computer. They combine ideas from classical information theory, computer science, and quantum physics. Instead of storing information in bits as in conventional digital computers do, quantum computers use quantum bits or quabits to encode information. Some believe that quantum computers hold the key to true artificial intelligence [4]. The main problem with quantum nanocomputer is instability.

Leading companies such as IBM, Intel, Motorola, and HP are investing heavily in research and development of nanocomputers.

\section{APPLICATIONS OF NANOCOMPUTING}

Nanocomputing will allow for the solution of complex real world problems. DNA nanocomputers is suited for application in data processing and can produce faster problem solution through the ability to explore all potential solutions simultaneously.

Common applications of nanocomputing include [5]:

- DNA Nanocomputing: Nanocomputing can be delivered by various nanoscale structures including biomolecules, for example, DNA and proteins. Practical applications of nanocomputing will require the ability to control and program DNA flexibly.

- Quantum Computing: A nanocomputer is sometimes called a quantum computer. Quantum computing provides computational power at the nanoscale with abilities that reach beyond the limitations of conventional computers. There are many different ways to instantiate quantum computing

- Nanorobot, or Nanobots: These will be controlled and managed by nanocomputers. Nano robot can be used for conducting complicated nano surgery on living cells and bacteria. This is possible because of the accurate position control of the nano robot. The nano robot is capable of imaging, manipulation, measuring mechanical properties, and tracking [6].

\section{BENEFITS AND CHALLENGES}

Nanocomputing has proved its usefulness in almost every branch of science and engineering. It has the unique advantage of being created to fit into any environment, including the human bod. With nanotechnology, it is possible to tailor the structures of materials at extremely small scales to achieve specific properties. Electronics has greatly benefited from nanotechnology, leading 
to faster, smaller, and more portable systems that can store large amount of data. Nanomedicine is an application of nanotechnology in medicine that produces precise solutions for disease prevention, diagnosis, and treatment. Nanotechnology offers several means of improving the transportation infrastructure [7].

As nanocomputing evolves, it will be feasible to have more complex and more tightly integrated systems.

Building systems at the atomic scale faces serious challenges. What will nanocomputers "look" like? Upon what operating principles will they function? Do we

need drastic new approaches to design hardware and software? How will individual devices be connected together? Once designed, how will these computers be fabricated? One challenge is to build a nanoscale memory which can be integrated with existing electronics. A nanoscale network router has the challenge of incorporating high bandwidth $\mathrm{I} / \mathrm{O}$ at the nanoscale. Embedded controllers are sensitive to power, price, volume, performance, and reliability [3,8]. When assembling different nanoobjects, their locations cannot be controlled. These challenges must be addressed for most nanotechnologies before they can replace conventional CMOS.

\section{CONCLUSION}

Nanocomputing technology has the potential to revolutionize the way that computers are used. Future nanocomputers could be evolutionary, working basically in the same ways as today's computers, but with nanoscale devices. Nanocomputing is still in its infancy.

It is increasingly clear that nanotechnology and nanocomputing will greatly impact the careers of many of our graduate students. Therefore, it is expedient to let graduate students have solid exposure to nanocomputing so that they will be better prepared to solve future problems. More information about nanocomputing can be found in books in [9-13] and many others available in Amazon.com.

\section{REFERENCES}

[1] R. Yadav, C. K. Dixit, and S. K. Trivedi, "Nanotechnology and nano computing,"

International Journal for Research in Applied Science \& Engineering Technology, vol. 5, no. X, October 2017.

[2] M. N.O. Sadiku, M. Tembely, and S.M. Musa, "Nanotechnology: An introduction," International Journal of Software and Hardware Research in Engineering, vol. 4, no. 5, May 2016, pp. 40-44.

[3] M. S. Montemerlo et al., "Technologies and designs for electronic nanocomputers," The MITRE Corporation, 1996, https://www.mitre.org/sites/default/files/pdf/96W0160.pdf

[4] M. N. O. Sadiku, M. Tembely, and S.M. Musa, "Quantum computing: A primer," International Journal of Advanced Research in Computer Science and Software Engineering, vol. 7, no. 11, Nov. 2017, pp. 129-130.

[5] J. M. Chatterjee and S. Dewangan, "Nanocomputing \& its future trends,"

International Journal of Advanced Computational Engineering and Networking, vol. 5, no. 11, Nov. 2017.

[6] B. Song et al., "Cellular-level surgery using nano robots," Journal of Laboratory Automation, vol. 17. no. 6, 2012, pp. 425434.

[7] "Benefits and applications,"

https://www.nano.gov/you/nanotechnology-benefits

[8] "NanoComputing," unknown source.

[9] S. K. Shukla and R. I. Bahar (eds.), Nano, Quantum and Molecular Computing: Implications to High Level Design and Validation. Springer, 2004.

[10] N. G. Anderson and S. Bhanja (eds.), Field-Coupled Nanocomputing:

Paradigms, Progress, and Perspectives. Springer, 2014.

[11] J. B. Waldner, Nanocomputers and Swarm Intelligence. Wiley-ISTE, 2013.

[12] J. Hsu, Nanocomputing: Computational Physics for Nanoscience and Nanotechnology. River Edge, NJ:

World Scientific Publishing, 2009.

[13] D. Tyagi, Nanocomputing. Shree Publishers, 2013.

\section{ABOUT THE AUTHORS}

Matthew N.O. Sadiku (sadiku@iee.org) is a professor at Prairie View A\&M University, Texas. He is the author of several books and papers. He is an IEEE fellow. His research interests include computational electromagnetics and computer networks. 
International Journal of Advances in Scientific Research and Engineering (ijasre), Vol 5 (3), March-2019

Chandra M. M. Kotteti (ckotteti@student.pvamu.edu) is currently a doctoral student at Prairie View A\&M University, Texas. His research interests include fake news detection using machine learning and deep learning, natural language processing, big data analytics, and wireless networks.

Sarhan M. Musa (smmusa@pvamu.edu) is a professor in the Department of Engineering Technology at Prairie View A\&M University, Texas. He has been the director of Prairie View Networking Academy, Texas, since 2004. He is an LTD Sprint and Boeing Welliver Fellow. 